EUROPEAN ORGANIZATION FOR NUCLEAR RESEARCH

CERN - AB Division

CERN-AB-2003-016 ABP

\title{
Adiabatic Capture of Charged Particles in Stable Islands: a Novel Approach to Multi-Turn Extraction
}

R. Cappi, M. Giovannozzi

\begin{abstract}
Recently a novel approach has been proposed aimed at performing multi-turn extraction from a circular machine. Such an approach consists of splitting the beam by means of stable islands created in transverse phase space by nonlinear magnetic elements such as sextupoles and octupoles. Provided a slow time-variation of the linear tune is applied, adiabatic with respect to the betatron motion, the islands can be moved in phase space and eventually charged particles may be trapped inside the stable structures. This generates a certain number of well-separated beamlets. Originally, this principle was successfully tested using a fourth-order resonance. In this paper the approach is generalised to other type of resonances and some examples of adiabatic capture performed by using various low-order resonances are presented and described in detail.
\end{abstract}

Presented at PAC 2003, Portland, Oregon, USA

from 12 to 16 May 2003

Geneva, Switzerland

22 May 2003 


\title{
ADIABATIC CAPTURE OF CHARGED PARTICLES IN STABLE ISLANDS: A NOVEL APPROACH TO MULTI-TURN EXTRACTION
}

\author{
R. Cappi and M. Giovannozzi, CERN, Geneva, Switzerland
}

\begin{abstract}
Recently a novel approach has been proposed aimed at performing multi-turn extraction from a circular machine. Such an approach consists of splitting the beam by means of stable islands created in transverse phase space by nonlinear magnetic elements such as sextupoles and octupoles. Provided a slow time-variation of the linear tune is applied, adiabatic with respect to the betatron motion, the islands can be moved in phase space and eventually charged particles may be trapped inside the stable structures. This generates a certain number of well-separated beamlets. Originally, this principle was successfully tested using a fourthorder resonance. In this paper the approach is generalised to other type of resonances and some examples of adiabatic capture performed by using various low-order resonances are presented and described in detail.
\end{abstract}

\section{INTRODUCTION}

A special technique is normally used to fill the CERN Super Proton Synchrotron (SPS) with a high-intensity proton beam from the Proton Synchrotron (PS), the so-called Continuous Transfer (CT) [1]. The beam is sliced using an electrostatic septum: by properly defining a closed orbit perturbation and by setting the horizontal tune equal to $Q_{H}=6.25$ the beam is extracted from the PS over five consecutive turns. A continuous ribbon four-turn long is extracted first, while the remaining central core is extracted last thus generating a proton spill five-turn long (see Refs. [1, 2] for more details). Such a multi-turn extraction might not be the best choice for the planned CERN Neutrino to Gran Sasso (CNGS) proton beam [3], especially in case of an intensity upgrade [4], due to the intrinsic losses (of the order of $15-20 \%$ of the overall proton intensity) onto the electrostatic septum as well as the poor betatron matching of the resulting slices.

An alternative method was proposed [5, 6]. The beam is split in the transverse horizontal phase space by the combined effect of a slow tune variation and the presence of stable islands created by nonlinear elements: the beam is trapped inside stable islands, creating a number of beamlets corresponding to the order of the resonance used. For the specific CERN application the fourth-order resonance was studied [5]. Following the encouraging results, numerical simulations were performed also for the third-order resonance [6] showing the validity of the method even in that case.

Preliminary experimental results obtained during the 2002 run of the PS machine allowed observing the trapping process into stable islands of phase space in real machines [7].
In this paper the latest results of numerical simulations are presented, showing how to perform multi-turn extraction for a small number of turns $(2-6)$ using adiabatic trapping inside a properly chosen resonance.

\section{NUMERICAL MODEL}

By assuming that the nonlinear magnets are located at the same place in the ring, that they are represented in the single-kick approximation [9], and that the vertical motion can be neglected, the one-turn transfer map can be expressed as $\hat{\mathbf{X}}_{n+1}=\mathbf{M}_{n}\left(\hat{\mathbf{X}}_{n}\right)$ :

$$
\left(\begin{array}{c}
\hat{X}_{n+1} \\
\hat{X}_{n+1}^{\prime}
\end{array}\right)=R\left(2 \pi \nu_{n}\right)\left(\begin{array}{c}
\hat{X}_{n} \\
\hat{X}_{n}^{\prime}+\hat{X}_{n}^{2}+\kappa \hat{X}_{n}^{3}
\end{array}\right),
$$

where $\left(\hat{X}, \hat{X}^{\prime}\right)$ are obtained from the Courant-Snyder coordinates [8] $\left(\hat{x}, \hat{x}^{\prime}\right)$ by means of the non-symplectic transformations [9]

$$
\left(\hat{X}, \hat{X}^{\prime}\right)=\frac{K_{2} \beta_{H}^{3 / 2}}{2}\left(\hat{x}, \hat{x^{\prime}}\right) \quad K_{l}=\frac{L}{B_{0} \rho} \frac{\partial^{l} B_{y}}{\partial x^{l}},
$$

$K_{2}\left(K_{3}\right)$ being the integrated sextupole (octupole) strength, $L$ the length of the nonlinear element, $B_{y}$ the vertical component of the magnetic field, $B_{0} \rho$ the magnetic rigidity, and $\beta_{H}$ the value of the horizontal beta-function at the location of the nonlinear elements. $R\left(2 \pi \nu_{n}\right)$ is a $2 \times 2$ rotation matrix of angle $\nu_{n}$, the fractional part of $Q_{H}$, and $\kappa$ is expressed as

$$
\kappa=\frac{2}{3} \frac{K_{3}}{\beta_{H} K_{2}^{2}}
$$

\section{RESULTS OF NUMERICAL SIMULATIONS}

\section{Two-Turn Extraction}

The case of a two-turn extraction is considered first, as it deserves special consideration. In fact, the map (1) does not possess any stable islands related to a resonance of order two (more details on this point can be found in Ref. [10]). To overcome this difficulty, a solution consists in assuming that the sextupoles and octupoles are not placed in the same location in the ring. In the numerical simulations they have been located $\pi / 2$ apart in betatron phase advance. Figure 1 reports the tune as a function of turn number. The four points labelled with letters refer to the tune values at which the beam distribution is shown in Fig. 2. The adiabatic capture inside the two stable islands is clearly visible. A small fraction of particles remains near the origin of phase space, 


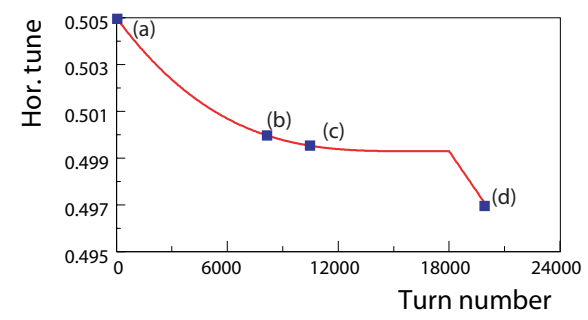

Figure 1: Evolution of the tune for a two-turn extraction.

while the majority of them migrates to the islands as the tune is swept through the half-integer resonance. Once the two beamlets are created, it is possible to move the islands towards higher-amplitudes, hence increasing the beamlets separation so to prepare for extraction.
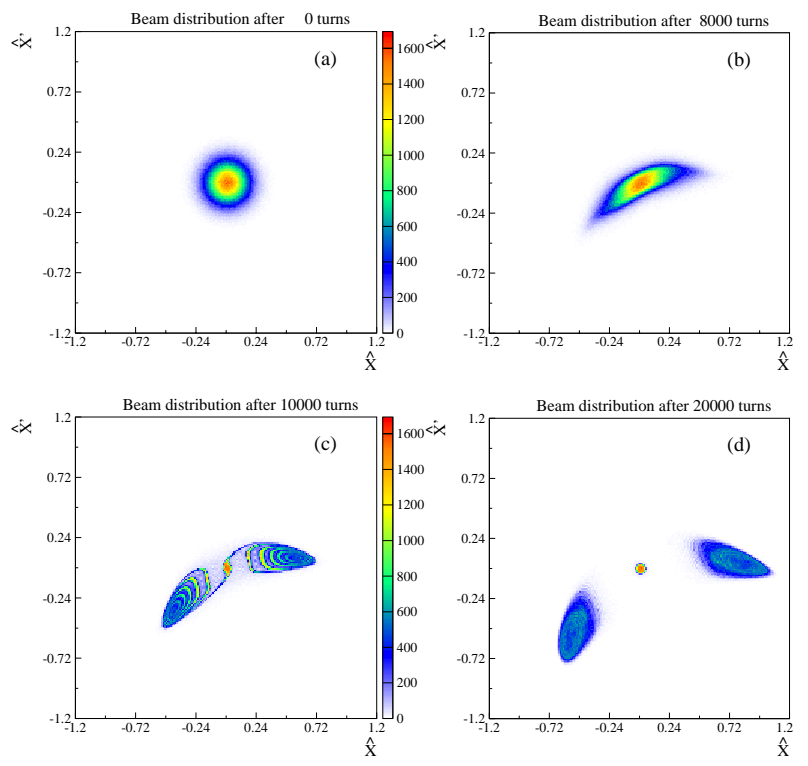

Figure 2: Beam distribution during the trapping process with two islands $\left(8.1 \times 10^{5}\right.$ initial condition). The initial distribution is a Gaussian centred on zero, with a standard deviation $\sigma=0.11$. Only a few particles are left near the origin of phase space.

\section{Three-Turn Extraction}

In this case the model (1) is used in the numerical simulations without any modification. The time-evolution of the linear tune is presented in Fig. 3, while the evolution of the beam distribution is shown in Fig. 4. The fact that

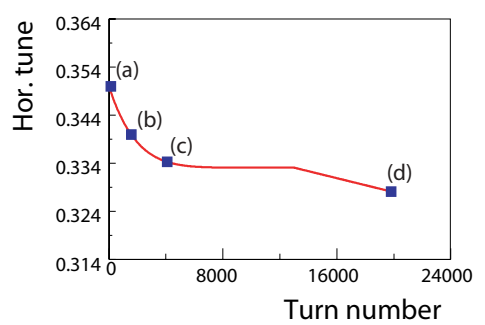

Figure 3: Evolution of the tune for a three-turn extraction. the resonance is intrinsically unstable makes it possible to deplete almost perfectly the region near the origin of phase space, thus generating three beamlets as a final result of the capture process.
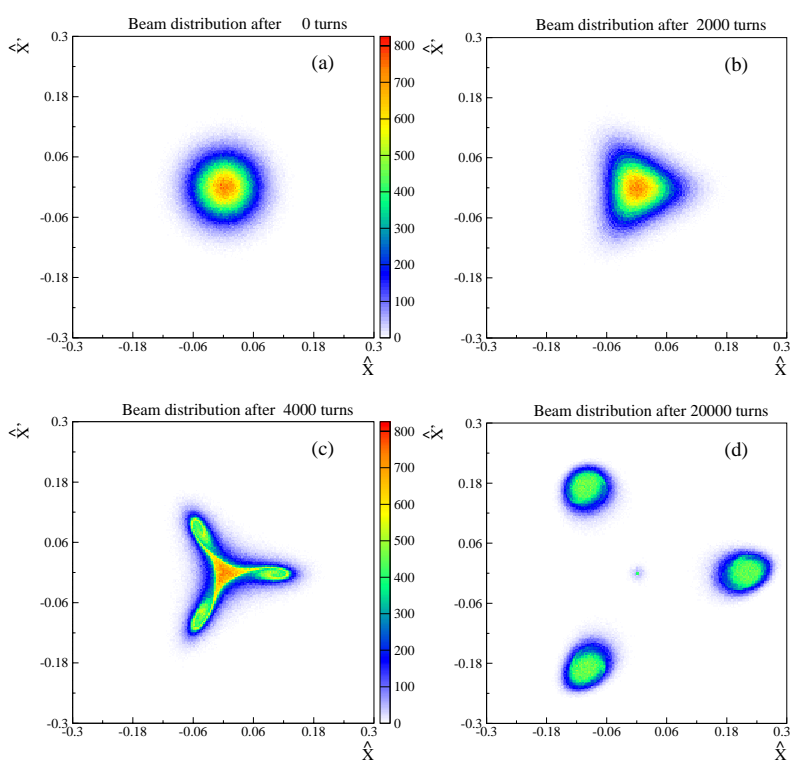

Figure 4: Beam distribution during the trapping process with three islands $\left(8.1 \times 10^{5}\right.$ initial conditions $)$. The initial distribution is a Gaussian centred on zero, with standard deviation $\sigma=0.04$. Even in this case very few particles are left in the central part of phase space.

\section{Five-Turn Extraction}

The fourth-order resonance is the one used in the original proposal [5] for a novel multi-turn extraction to replace the present CT extraction mode. As the fourth-order resonance is stable for the system (1), the beam will be split into five beamlets. The evolution of the beam distribution is plotted

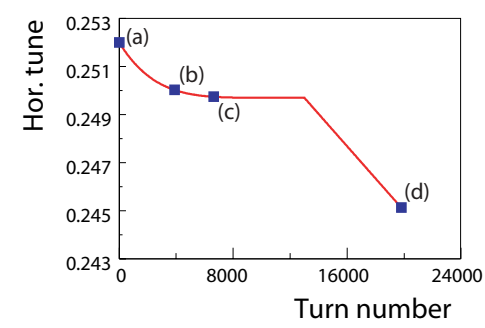

Figure 5: Evolution of the tune for a five-turn extraction.

in Fig. 6. The four outermost beamlets are created by adiabatic trapping inside the stable islands, while the fifth one is generated by those particles left unperturbed near the origin of phase space. This fact has deep implications on the properties of the beamlets. In fact, for the previous multiturn extraction processes (two- and three-turn) the various beamlets had the same number of particles trapped inside as they represent a unique ribbon of length two or three times the machine circumference. However, when the central part of phase space is filled at the end of the capture process, the beamlets need not be equally populated, unless a dedicated optimisation of the free parameters is performed. 
Such an optimisation concerns mainly the sigma of the initial beam distribution, the nonlinear elements strength, and the properties of the tune-variation.
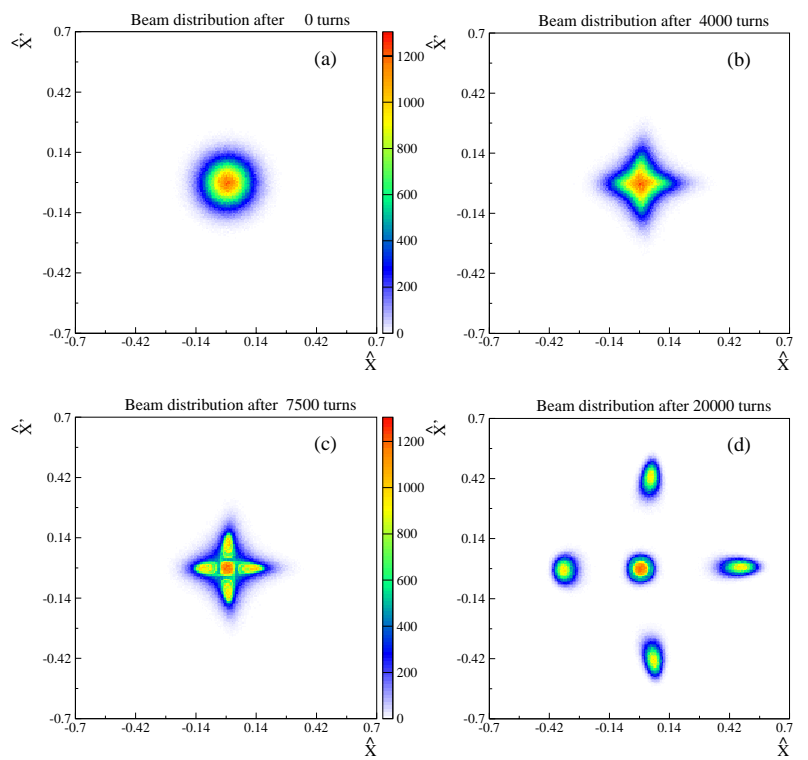

Figure 6: Beam distribution during the trapping process with four islands $\left(8.1 \times 10^{5}\right.$ initial conditions). The initial distribution is a Gaussian centred on zero, with standard deviation $\sigma=0.073$.

\section{Six-Turn Extraction}

By using even higher-order resonances it is possible to increase further the length of the extracted spill. Here, the case of a resonance of order five is shown in Figs. 7 and 8. However, it is clear that there are intrinsic limitations on the resonance order. In fact, the higher the order, the smaller will be the islands, thus enhancing the difference between the first turns and the last one, represented by the particles left around the origin in phase space at the end of the capture process. Furthermore, it will be more and more difficult to create enough free space between the beamlets to accommodate the blade of an extraction septum.

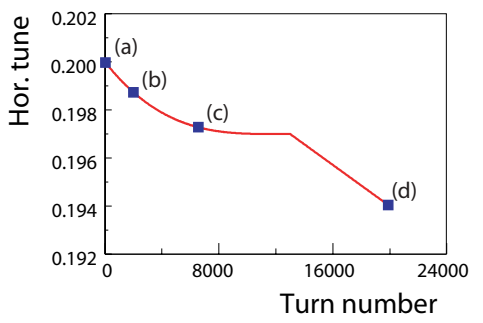

Figure 7: Evolution of the tune for a six-turn extraction.

\section{CONCLUSIONS}

In this paper various schemes for multi-turn extraction based on adiabatic capture of charged particles in stable islands of phase space were presented. By choosing the appropriate resonance, two-, three-, five-, and six-turn extraction have been computed. In all the cases shown here, par- ticles can be trapped inside islands without any loss. Furthermore, it is possible to move the beamlets by acting on the tune so to increase their separation, which is the necessary condition to allow the actual beam extraction to take place. Further studies are in progress to quantify a number of issues such as the adiabaticity of the process and the dependence of the extracted beam parameters on the strength of the nonlinear elements.
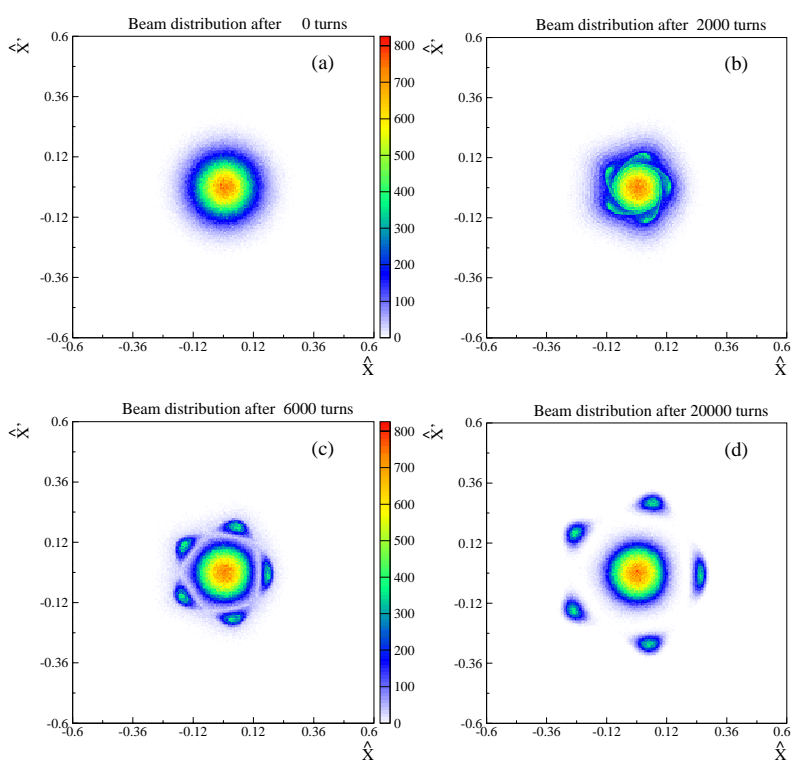

Figure 8: Beam distribution during the trapping process with five islands $\left(8.1 \times 10^{5}\right.$ initial conditions). The initial distribution is a Gaussian centred on zero, with standard deviation $\sigma=0.08$.

\section{REFERENCES}

[1] C. Bovet et al., in 1973 Particle Accelerator Conference, edited by D. W. Dupen (IEEE, New York, 1973) p. 438.

[2] R. Cappi, M. Giovannozzi, "Computation of Betatron Mismatch and Emittance Blow-up for Multi-Turn Extraction", CERN PS (AE) 2002-083 (2002).

[3] K. Elsener (Ed.) et al., "The CERN Neutrino Beam to Gran Sasso (Conceptual Technical Design)”, CERN 98-02 (1998).

[4] R. Cappi (Ed.) et al., "Increasing proton intensity of PS and SPS”, CERN-PS (AE) 2001-041 (2001).

[5] R. Cappi, M. Giovannozzi, Phys. Rev. Lett. 88, (2002) 104801.

[6] R. Cappi, M.Giovannozzi, in Eighth Particle European Accelerator Conference, edited by J. Poole and C. Petit-JeanGenaz (Institute of Physics, UK London, 2002) p. 1250.

[7] R. Cappi et al., "Adiabatic Beam Trapping in Stable Islands of Transverse Phase Space: Measurement Results at CERN Proton Synchrotron", these proceedings.

[8] E. Courant and H. Snyder, Ann. Phys. 3, (1958) p. 1.

[9] A. Bazzani et al., CERN 94-02 (1994).

[10] R. Cappi, M. Giovannozzi, "Multi-turn Extraction and Injection by Means of Adiabatic Capture in Stable Islands of Phase Space", in preparation. 